\title{
ON EULER METHODS OF SUMMABILITY FOR DOUBLE SERIES
}

\author{
G. N. WOLLAN
}

The two $q$ th order Euler transforms of the sequence $A_{n}$

and

$$
A_{n}^{q}=(q+1)^{-n-1} \sum_{k=0}^{n}\left(\begin{array}{c}
n+1 \\
k+1
\end{array}\right) q^{n-k} A_{k}
$$

$$
B_{n}^{q}=(q+1)^{-n} \sum_{k=0}^{n}\left(\begin{array}{l}
n \\
k
\end{array}\right) q^{n-k} A_{k}
$$

are equivalent for $q \geqq 0$ in the sense that if either has a limit as $n \rightarrow \infty$ the other has the same limit $\left[1\right.$, p. 180]. ${ }^{1}$ For double sequences the corresponding transforms are

$$
\begin{aligned}
& A_{m n}^{q}=(q+1)^{-m-n-2} \sum_{h, k=0}^{m, n}\left(\begin{array}{c}
m+1 \\
h+1
\end{array}\right)\left(\begin{array}{c}
n+1 \\
k+1
\end{array}\right) q^{m+n-h-k} A_{k k}, \\
& B_{m n}^{q}=(q+1)^{-m-n} \sum_{k, k=0}^{m, n}\left(\begin{array}{c}
m \\
h
\end{array}\right)\left(\begin{array}{l}
n \\
k
\end{array}\right) q^{m+n-h-k} A_{h k .} .
\end{aligned}
$$

This paper is concerned with two theorems regarding these transforms. Throughout the discussion $q \geqq 0$.

TheOREM 1. If $A_{m=}^{e}$ has a limit as $m, n \rightarrow \infty$, then $B_{m=n}^{e}$ has that same limit and if $B_{m m}$ has a limit and is bounded, then $A_{m m}^{e_{m}}$ has that same limit but there do exist sequences for which $B_{\text {man }}$ has a limit but for which $\lim _{m, n \rightarrow \infty} A_{m}$ does not exist for any $q \geqq 0$.

The relation

$$
B_{m n}^{q}=q^{2} A_{m-1, n-1}^{q}-q(q+1)\left(A_{m, n-1}^{q}+A_{m-1, n}^{q}\right)+(q+1)^{2} A_{m n}^{q}
$$

may be verified by substitution from (1) into the right-hand side. This relation may be written in the form

$$
\begin{aligned}
B_{m n}^{q}= & q^{2}\left(A_{m-1, n-1}^{q}-A_{m, n-1}^{q}-A_{m-1, n}^{q}+A_{m n}^{q}\right) \\
& -q\left(A_{m, n-1}^{q}+A_{m-1, n}^{q}-2 A_{m n}^{q}\right)+A_{m n}^{q} .
\end{aligned}
$$

Presented to the Society, November 29, 1952; received by the editors December 4, 1952.

1 Numbers in brackets refer to the references at the end of the paper. 
From this relation (4) it follows that if $\lim _{m, n \rightarrow \infty} A_{m n}^{q}=A$, then $\lim _{m, n \rightarrow \infty} B_{m n}^{q}=A$.

Relation (3) can be used to express $A_{m m}^{q}$ in terms of $B_{m n}^{q}$. First write (3) in the form

$$
(q+1)^{2} A_{m n}^{q}=B_{m n}^{q}+q(q+1)\left(A_{m, n-1}^{q}+A_{m-1, n}^{q}\right)-q^{2} A_{m-1, n-1}^{q} .
$$

In this replace $A_{m, n-1}^{q}$ and $A_{m-1, n}^{q}$, by the values which this relation gives for them. This yields

$$
\begin{aligned}
(q+1)^{2} A_{m n}^{q}= & B_{m n}^{q}+q(q+1)^{-1}\left(B_{m, n-1}^{q}+B_{m-1, n}^{q}\right) \\
& +q^{2}\left(A_{m, n-2}^{q}+A_{m-1, n-1}^{q}+A_{m-2, n}^{q}\right) \\
& -q^{3}(q+1)^{-1}\left(A_{m-1, n-2}^{q}+A_{m-2, n-1}^{q}\right) .
\end{aligned}
$$

Successive repetitions of this procedure lead finally to the relation

$$
(q+1)^{2} A_{m n}^{q}=\sum_{h, k=0}^{m, n}\left(\frac{q}{q+1}\right)^{m+n-h-k} B_{h k}^{q} .
$$

Relation (5) expresses $A_{m n}^{\mathfrak{q}}$ as a transform of the sequence $B_{m n}^{q}$. The coefficients of the transformation satisfy the conditions for regularity [3, p. 23]. Hence if $B_{m n}^{q}$ has the limit $A$ and is bounded, then $A_{m n}^{q}$ also has the limit $A$.

To see that there exist sequences for which the transform $B_{m n}^{a}$ has a limit but for which $\lim _{m, n \rightarrow \infty} A_{m n n}^{q}$ does not exist for any $q \geqq 0$ consider the sequence $A_{m n}=(-1)^{n} p^{2 m+n-1}\{n(p+1)+p\}, p>1$. For this sequence one may readily verify by substitution into (2) that $B_{m n}^{p}=0$ whenever $n>1$. Thus for this sequence the transform $B_{m n}^{p}$ has the limit 0 . But by substituting into (1) and simplifying one obtains

$$
\begin{aligned}
A_{m n}^{q}= & \left\{\left(\frac{q+p^{2}}{q+1}\right)^{m+1}-\left(\frac{q}{q+1}\right)^{m+1}\right\} \\
& \cdot\left(\frac{n+1}{q+1}\right) \cdot\left(\frac{p+1}{p^{3}}\right) \cdot\left(\frac{q-p}{q+1}\right)^{n} \\
& +p^{-3}\left\{\left(\frac{q+p^{2}}{q+1}\right)^{m+1}-\left(\frac{q}{q+1}\right)^{m+1}\right\} \\
& \left\{\left(\frac{q-p}{q+1}\right)^{n+1}-\left(\frac{q}{q+1}\right)^{n+1}\right\}
\end{aligned}
$$

and for $p>1$ this does not have a limit for any $q \geqq 0$. This completes the proof of Theorem 1 . 
THEOREM 2. Let $A_{m n}=\sum_{h, k=0}^{m, n} a_{h k}$. If

$$
\text { (1) } B_{m n}^{1}=2^{-m-n} \sum_{h, k=0}^{m, n}\left(\begin{array}{l}
m \\
h
\end{array}\right)\left(\begin{array}{l}
n \\
k
\end{array}\right) A_{h k}
$$

has the limit $A$ as $m, n \rightarrow \infty$, (2) $A_{m n}$ is bounded and

$$
\text { (3) } \lim _{m, n \rightarrow \infty}\left(m^{1 / 2}+n^{i / 2}\right)(m n)^{1 / 2} a_{m n}=0 \text {, }
$$

then $A_{m n}$ also has the limit $A$ as $m, n \rightarrow \infty$.

Form the difference

$$
B_{4 m, 4 n}^{1}-A_{2 m, 2 n}=2^{-4 m-4 n} \sum_{h, k=0}^{4 m, 4 n}\left(\begin{array}{c}
4 m \\
h
\end{array}\right)\left(\begin{array}{c}
4 n \\
k
\end{array}\right)\left(A_{h k}-A_{2 m, 2 n}\right) .
$$

Separate this difference into 9 parts $S_{1}, S_{2}, \cdots, S_{9}$ corresponding respectively to the intervals of summation

$$
\begin{array}{lll}
\left(\begin{array}{c}
0 \leqq h \leqq m \\
0 \leqq k \leqq n
\end{array}\right), & \left(\begin{array}{l}
0 \leqq h \leqq m \\
n<k<3 n
\end{array}\right), & \left(\begin{array}{c}
0 \leqq h \leqq m \\
3 n \leqq k \leqq 4 n
\end{array}\right), \\
\left(\begin{array}{c}
m<h<3 m \\
0 \leqq k \leqq n
\end{array}\right), & \left(\begin{array}{l}
m<h<3 m \\
n<k<3 n
\end{array}\right), & \left(\begin{array}{c}
m<h<3 m \\
3 n \leqq k \leqq 4 n
\end{array}\right), \\
\left(\begin{array}{c}
3 m \leqq h \leqq 4 m \\
0 \leqq k \leqq n
\end{array}\right), & \left(\begin{array}{c}
3 m \leqq h \leqq 4 m \\
n<k<3 n
\end{array}\right), & \left(\begin{array}{c}
3 m \leqq h \leqq 4 m \\
3 n \leqq k \leqq 4 n
\end{array}\right) .
\end{array}
$$

Since

$$
\begin{gathered}
2^{-4 m} \sum_{h=0}^{4 m}\left(\begin{array}{c}
4 m \\
h
\end{array}\right)=1, \\
\lim _{m \rightarrow \infty} 2^{-4 m} \sum_{h=0}^{m}\left(\begin{array}{c}
4 m \\
h
\end{array}\right)=0[2, \text { p. } 511], \quad \lim _{m \rightarrow \infty} 2^{-4 m} \sum_{h=3 m}^{4 m}\left(\begin{array}{c}
4 m \\
h
\end{array}\right)=0,
\end{gathered}
$$

and $A_{m n}$ is bounded it follows that each of the parts $S_{1}, S_{2}, S_{3}, S_{4}$, $S_{6}, S_{7}, S_{8}, S_{9}$ has the limit zero as $m, n \rightarrow \infty$. Thus if $S_{5}$ has the limit zero it will follow that the difference $B_{4 m, 4 n}^{1}-A_{2 m, 2 n}$ has the limit zero.

Let $Q_{m, n}$ denote the largest of the numbers $\left((m+h)^{1 / 2}+(n+k)^{1 / 2}\right)$ $\cdot((m+h)(n+k))^{1 / 2} \cdot\left|a_{m+h, n+k}\right|$ for $m<h<3 m$ and $n<k<3 n$. Then for all $h, k$ in these intervals

$$
\begin{aligned}
& \left|A_{h k}-A_{2 m, 2 n}\right| \\
& \quad \leqq(|2 m-h| \cdot 3 n+|2 n-k| \cdot 2 m) \frac{Q_{m n}}{\left(m^{1 / 2}+\dot{n}^{1 / 2}\right)(m n)^{1 / 2}}
\end{aligned}
$$


if $m n \neq 0$. Hence

$$
\begin{aligned}
\left|S_{b}\right| \leqq & 2^{-4 m-4 n} \sum_{k, k=m+1, n+1}^{3 m-1,2 n-1}\left(\begin{array}{c}
4 m \\
k
\end{array}\right)\left(\begin{array}{c}
4 n \\
k
\end{array}\right)(|2 m-h| \cdot 3 n \\
& +|2 n-k| \cdot 2 m) \frac{Q_{m n}}{\left(m^{1 / 2}+n^{1 / 2}\right) \cdot(m n)^{1 / 2}} \\
\leqq & \left\{3 n \cdot 2^{-4 m} \sum_{n=m+1}^{3 m-1}|2 m-h| \cdot\left(\begin{array}{c}
4 m \\
k
\end{array}\right)\right. \\
& \left.+2 m \cdot 2^{-4 n} \sum_{k=n+1}^{3 n-1}|2 n-k| \cdot\left(\begin{array}{c}
4 n \\
k
\end{array}\right)\right\} \frac{Q_{m n}}{\left(m^{1 / 2}+n^{1 / 2}\right) \cdot(m n)^{1 / 2}} .
\end{aligned}
$$

But

$$
\sum_{h=m+1}^{8 m-1}|2 m-h| \cdot\left(\frac{4 m}{h}\right)<2 \sum_{h=0}^{2 m}(2 m-h)\left(\begin{array}{c}
4 m \\
h
\end{array}\right)
$$

and

$$
\begin{aligned}
\sum_{h=0}^{2 m}(2 m-h) & \left(\begin{array}{c}
4 m \\
h
\end{array}\right) \\
& =2 m\left\{\frac{1}{2} \sum_{h=0}^{4 m}\left(\begin{array}{c}
4 m \\
h
\end{array}\right)+\frac{1}{2}\left(\begin{array}{c}
4 m \\
2 m
\end{array}\right)\right\}-4 m \sum_{h=1}^{2 m}\left(\begin{array}{c}
4 m-1 \\
h-1
\end{array}\right) \\
& =m\left\{24 m+\left(\begin{array}{c}
4 m \\
2 m
\end{array}\right)-4 \sum_{h=0}^{2 m-1}\left(\frac{4 m-1}{h}\right)\right\}=m\left(\frac{4 m}{2 m}\right) .
\end{aligned}
$$

Hence

$$
\left|S_{b}\right|<\left\{6 m n \cdot 2^{-4 m}\left(\begin{array}{l}
4 m \\
2 m
\end{array}\right)+4 m n \cdot 2^{-4 n}\left(\begin{array}{l}
4 n \\
2 n
\end{array}\right)\right\} \frac{Q_{m n}}{\left(m^{1 / 2}+n^{1 / 2}\right) \cdot(m n)^{1 / 2}} .
$$

Since

$$
2^{-2 n}\left(\begin{array}{c}
2 n \\
n
\end{array}\right) \cong(\pi n)^{-1 / 2}
$$

$[2$, p. 385] it then follows that

$$
\begin{aligned}
\left|S_{6}\right|<\left\{6 m n(2 \pi m)^{-1 / 2}\right. & \left(1+e_{m}\right) \\
& \left.+4 m n(2 \pi n)^{-1 / 2}\left(1+e_{n}\right)\right\} \frac{Q_{m n}}{\left(m^{1 / 2}+n^{1 / 2}\right) \cdot(m n)^{1 / 2}}
\end{aligned}
$$

where $e_{m} \rightarrow 0$ as $m \rightarrow \infty$ and $e_{n} \rightarrow 0$ as $n \rightarrow \infty$. Thus 


$$
\left|S_{b}\right|<\left\{\frac{6 n^{1 / 2}\left(1+e_{m}\right)+4 m^{1 / 2}\left(1+e_{n}\right)}{m^{1 / 2}+n^{1 / 2}}\right\} \cdot Q_{m n} .
$$

Since the quantity in braces is bounded and $Q_{m n} \rightarrow 0$ it then follows that $S_{5} \rightarrow 0$ as $m, n \rightarrow \infty$. Hence the difference $B_{m, m}^{1}-A_{2 m, 2 n}$ has the limit zero. With only slight modifications of this argument it can be shown that $B_{4 m, 4 n}^{1}-A_{2 m+1,2 n}, B_{4 m, 1 n}^{1}-A_{2 m, 2 n+1}$, and $B_{4 m, 4 n}^{1}-A_{2 m+1,2 n+1}$ have the limit zero. The proof of the theorem is then complete.

\section{REFERENCES}

1. G. H. Hardy, Divergeñt series, Oxford, 1949.

2. K. Knopp, Theory and applications of infinite series, London, 1928.

3. C. N. Moore, Summable series and convergence factors, Amer. Math. Soc. Colloquium Publications, vol. 22, 1938.

Memphis State College 